\title{
Expanding horizons in metastatic prostate cancer treatment
}

\section{Karim Fizazi and Carsten Goessl}

We read with interest the recent, comprehensive review by Sturge et al. (Bone metastasis in prostate cancer: emerging therapeutic strategies. Nat. Rev. Clin. Oncol. 8, 357-368) ${ }^{1}$ that describes current and emerging therapies for bone metastases in patients with castration-resistant prostate cancer (CRPC). However, we noted several factual errors and additional points that we consider should be further clarified.

The authors claim an overall survival benefit for clodronate treatment in men with bone metastases from CRPC on the basis of the data from the Medical Research Council (MRC) PR05 trial. ${ }^{2}$ However, patients with bone metastases enrolled in this trial did not have castration-resistant disease. Furthermore, as overall survival was not reported in the entire cohort in the MRC PR05 trial, it is difficult to draw definitive conclusions on the impact of clodronate on survival in men with metastatic prostate cancer.

Sturge et al. ${ }^{1}$ refer to the approval of denosumab in November of 2010 for prevention of skeletal-related events in patients with bone metastases from solid tumors and conclude in the 'key points' box that the efficacy of denosumab in metastatic bone disease (MBD) associated with CRPC is unclear. We appreciate that the full publication of the head-to-head trial of denosumab versus zoledronic acid in patients with bone metastases from CRPC $^{3}$ was not available to Sturge et al. ${ }^{1}$ at the time their manuscript was submitted. However, the trial had been previously reported at the ASCO 2010 meeting, ${ }^{4}$ and is also described in the US denosumab package insert, which was publicly available once approval was obtained. There is evidence from a phase III, large, randomized, double-blind, controlled clinical trial that denosumab is not only efficacious for prevention of skeletal-related events in patients with bone metastases from CRPC, but also significantly superior to zoledronic acid. ${ }^{3}$ In addition, denosumab is a fully human monoclonal antibody, not a humanized antibody. This is important because fully human monoclonal antibodies are less likely to elicit an immune response. ${ }^{5}$ In addition, in opposition to the statement by Sturge et al., ${ }^{1}$ prevention of bone loss did not form the basis for the approval of zoledronic acid in the treatment of metastatic CRPC. To date, no bisphosphonate has been approved for the treatment of bone loss due to androgen-deprivation therapy in prostate cancer.

Finally, there are several factual errors related to Tables 1 and 2 . The title for these tables states that these studies are ongoing when in fact some of the listed trials are completed (for example, NCT00104650'6). Unlike claimed by Sturge et al., ${ }^{1}$ study NCT003307597 excluded prostate cancer patients (Table 1). Patients enrolled in trial NCT000896748 (Table 2) not only had an absence of bone metastases, as indicated by the authors, but did not present with any kind of metastases. In addition, fractures were a secondary end point in this trial, instead of skeletal-related events as noted. The final results of that study were published by Smith et al. ${ }^{9}$ in 2009 . Furthermore, trial NCT00321620 ${ }^{10}$ (Table 2) did not evaluate denosumab in addition to zoledronic acid as indicated, but rather compared denosumab head-to-head with zoledronic acid.,

The recent US approval of several new agents (sipuleucel-T, cabazitaxel, abiraterone and denosumab) has already changed the treatment landscape of metastatic CRPC and more agents are appearing on the horizon. Giving health-care providers an accurate representation of the available data is critical to maximize the benefit of these exciting developments for patients with metastatic CPRC.

Department of Cancer Medicine, Institut Gustave Roussy, University of Paris Sud,
39 rue Camille Desmoulins, Villejuif 94805 , France (K. Fizazi). Clinical Development, Amgen Inc., One Amgen Center Drive, MS 38-2-A, Thousand Oaks, CA 91320, USA (C. GoessI).

Correspondence to: K. Fizazi fizazi@igr.fr

\section{Acknowledgment}

Amy Foreman-Wykert (Amgen Inc.) assisted in draft preparation and formatting of the correspondence.

\section{Competing interests}

K. Fizazi declares associations with the following companies: Amgen, Novartis. C. Goessl declares an association with the following company: Amgen. See the article online for full details of the relationships.

1. Sturge, J., Caley, M. P. \& Waxman, J. Bone metastasis in prostate cancer: emerging therapeutic strategies. Nat. Rev. Clin. Oncol. 8, 357-368 (2011).

2. Dearnaley, D. P., Mason, M. D., Parmar, M. K., Sanders, K. \& Sydes, M. R. Adjuvant therapy with oral sodium clodronate in locally advanced and metastatic prostate cancer: long-term overall survival results from the MRC PRO4 and PR05 randomised controlled trials. Lancet Oncol. 10, 872-876 (2009).

3. Fizazi, K. et al. Denosumab versus zoledronic acid for treatment of bone metastases in men with castration-resistant prostate cancer: a randomised, double-blind study. Lancet. 377 , 813-822 (2011).

4. Fizazi, K. et al. A randomized phase III trial of denosumab versus zoledronic acid in patients with bone metastases from castration-resistant prostate cancer [abstract]. J. Clin. Oncol. 28 (Suppl. 18), LBA4507 (2010).

5. Weiner, L. M. Fully human therapeutic monoclonal antibodies. J. Immunother. 29, 1-9 (2006).

6. US National Library of Medicine. ClinicalTrials.gov [online], http://clinicaltrials.gov/ct2/show/ NCT00104650 (2011).

7. US National Library of Medicine. ClinicalTrials.gov [online], http://clinicaltrials.gov/ct2/show/ NCT00330759 (2010).

8. US National Library of Medicine. ClinicalTrials.gov [online], http://clinicaltrials.gov/ct2/show/ NCT00089674 (2009).

9. Smith, M. R. et al. Denosumab in men receiving androgen-deprivation therapy for prostate cancer. N. Engl. J. Med. 361, 745-755 (2009).

10. US National Library of Medicine. ClinicalTrials.gov [online], http://clinicaltrials.gov/ct2/show/ NCT00321620 (2010). 